\title{
VORTEX PERTURBATION DYNAMICS *
}

\author{
W.O. CRIMINALE \\ Department of Applied Mathematics \\ University of Washington \\ Seattle, Washington 98195 \\ D.G. LASSEIGNE \\ Department of Mathematics and Statistics \\ Old Dominion University \\ Norfolk, Virginia 23529 \\ T.L. JACKSON \\ Institute for Computer Applications in Science and Engineering \\ NASA Langley Research Center \\ Hampton, Virginia 23681
}

\begin{abstract}
An initial value approach is used to examine the dynamics of perturbations introduced into a vortex under strain. Both the basic vortex considered and the perturbations are taken as fully three-dimensional. An explicit solution for the time evolution of the vorticity perturbations is given for arbitrary initial vorticity. Analytical solutions for the resulting velocity components are found when the initial vorticity is assumed to be localized. For more general initial vorticity distributions, the velocity components are determined numerically. It is found that the variation in the radial direction of the initial vorticity disturbance is the most important factor influencing the qualitative behaviour of the solutions. Transient growth in the magnitude of the velocity components is found to be directly attributable to the compactness of the initial vorticity.
\end{abstract}

\footnotetext{
*This work was supported by the National Aeronautics and Space Administration under NASA Contract No. NAS1-19480 while in residence at the Institute for Computer Applications in Science and Engineering (ICASE), NASA Langley Research, Hampton, VA 23681-0001.
} 


\section{INTRODUCTION}

Vortex dynamics has been a significant subject of extensive investigation throughout the history of fluid mechanics. On the one hand, this interest has meant the actual establishment of the velocity compatible with a particular vortex with the results leading to numerous prototypical vortices that now carry names attributable to the person or the situation that created their existence. A Burgers vortex or a trailing vortex are but two of the numerous examples. Then, the question of the stability of any vortex has been asked. This is especially relevant if one wants to understand the consequences of a vortex under the influence of adverse or favorable strain. Finally, there is the topic of vortex breakdown, a special separate topic because of its consequences. Vorticity and vorticity interaction are the bases of turbulence. Heat and mass transfer rely to a large degree on vortex dynamics. And, there is the geophysical environment where vortices play major roles in both the ocean and the atmosphere. It is important, then, to know the behavior of vortices, particularly if they are subjected to external disturbances.

The use of classical stability theory in examining a vortex flow has met with only limited success due to the fact that the mean flow is always a function of more than one independent variable. As a result, traditional normal mode analysis can only be used in an approximate manner. (This conclusion is always inevitable if the perturbation system cannot be reduced to one of ordinary differential equations.) Consequently, an alternative method for analysis must be sought in order to understand the dynamics. One such procedure is available and has already been applied to one or more rotating flows or vortices in a plane. Here, since the basic mean flow can be synthesized from linear functions of the appropriate spatial variables (constant shear), more general solutions of the perturbation quantities can be obtained than those derived from normal modes. In addition, there is the bonus that the perturbation field can be considered three-dimensional ( $c$. Craik and Criminale ${ }^{1}$ for the basic method; Bayly ${ }^{2}$, Landman \& Saffman ${ }^{3}$, Waleffe ${ }^{4}$, Dritschell ${ }^{5}$, or Craik and Allen ${ }^{6}$, among others, for specific applications of the method to planar vortical or rotating flows). Both approaches have found that such basic flows can be unstable and the instability depends upon various parameters and whether or not the perturbations are two or three-dimensional. Regardless of the conclusions from any of these treatments, it should be stressed that the work has relied on a basic vortical velocity that is two-dimensional. More realistic vortices have flow along the axis of rotation as well as motion in the plane. In other words, the mean flow is three-dimensional and investigation of the stability should consider this fact along with the possibility of the perturbations being three-dimensional.

Any general treatment of the dynamics of perturbations in an arbitrary vortex is not possible analytically and is the principal reason why such work has been limited to or approximated in ways such as those cited above. Still, if one examines the family of vortices that is known and is willing to exchange one set of assumptions for others in order to obtain new insight, it is possible to investigate a three-dimensional vortex under three dimensional perturbations. This is the major point of this presentation. And, instead of seeking stability criteria per se, an initial-value problem is both posed and solved. The results are general, can be expressed in closed form, and both the early period transients as well as the asymptotic fate of disturbances can be determined explicitly. 


\section{BASIC PROBLEM}

One of the better references dealing with the derivation of vortical flow is that due to Donaldson and Sullivan ${ }^{7}$ where a great deal of effort was given to deriving a general solution for a vortex family. It was found that the basic mean velocity can be written as

$$
\vec{u}=\left(U_{r}(r), V_{\theta}(r), z W(r)\right)
$$

where the velocity components are given in the $r, \theta$, and $z$-directions respectively. The coordinates $(x, y)$ define the plane with $r=\left(x^{2}+y^{2}\right)^{1 / 2}, \theta=\tan ^{-1}(y / x) ; z$ is perpendicular to the plane of rotation. This representation contains many of the more well-known examples, such as the Burgers' vortex, Rott's modified Burgers' vortex, or the Batchelor trailing line vortex among others. In addition, (1) also contains viscous effects. The simplest fully three-dimensional vortex with this form of representation is the potential vortex under strain and is a limiting form of (1) or

$$
U_{r}=-\frac{\epsilon}{2} r, \quad V_{\theta}=\frac{\Gamma_{0}}{r}, \quad W=\epsilon .
$$

The coefficient $\Gamma_{0}$ is proportional to the circulation, and the coefficient $\epsilon$, which can be timedependent and even negative, is proportional to the strain rate. The perturbation problem associated with the vortex (2) can be solved explicitly as an initial-value problem. In effect, (2) is the inviscid limit of (1), and although being three-dimensional, it does not have a finite core. This lack in the basic flow does not carry over to the perturbation field. The pressure corresponding to $(2)$ is

$$
P=-\frac{\epsilon^{2}}{8}\left(r^{2}+4 z^{2}\right)-\frac{\Gamma_{0}}{2 r^{2}} .
$$

The vortex represented by (2) has zero vorticity. Thus, it is convenient to pose the perturbation problem in terms of the perturbation vorticity which is the leading term for the vorticity of the flow itself. With the additional assumptions of an incompressible and inviscid fluid, the perturbation equations for the vorticity are

$$
\begin{gathered}
\frac{D \omega_{r}}{D t}+\frac{\epsilon}{2} \omega_{r}=0, \\
\frac{D \omega_{\theta}}{D t}+\frac{\epsilon}{2} \omega_{\theta}+\frac{2 \Gamma_{0}}{r^{2}} \omega_{r}=0,
\end{gathered}
$$

and

$$
\frac{D \omega_{z}}{D t}-\epsilon \omega_{z}=0
$$

where the differential operator is given by

$$
\frac{D}{D t}=\frac{\partial}{\partial t}-\frac{\epsilon r}{2} \frac{\partial}{\partial r}+\frac{\Gamma_{0}}{r^{2}} \frac{\partial}{\partial \theta}+\epsilon z \frac{\partial}{\partial z} .
$$

The variables $\omega_{r}, \omega_{\theta}$ and $\omega_{z}$ are the vorticity components in the $r, \theta$ and $z$ directions, respectively. In this differential operator, the spatial variables $r$ and $z$ appear linearly as coefficients of the partial derivatives with respect to $r$ and $z$, allowing for a transformation of the independent variables to a moving coordinate frame given by

$$
\xi=e^{\frac{1}{2} \int_{0}^{t} \epsilon\left(t^{\prime}\right) d t^{\prime}} r=\psi_{1}(t) r
$$




$$
\zeta=e^{-\int_{0}^{t} \epsilon\left(t^{\prime}\right) d t^{\prime}} z=\psi_{2}(t) z
$$

and

$$
T=t
$$

In this frame, advection due to the mean flow in these directions is removed from (4)-(6). At the initial time $t=T=0$, the coordinates coincide as $\xi=r$ and $\zeta=z$, and there is no difficulty in applying initial conditions. Since the solution must be periodic in the $\theta$ variable on physical grounds, the transformation of the dependent variables as $f \rightarrow f e^{i n \theta}$ will be used throughout. On combining these two steps, the differential operator (7) reduces to

$$
\frac{D}{D t}=\frac{\partial}{\partial T}+\frac{i n \Gamma_{0} \psi_{1}^{2}}{\xi^{2}}
$$

and the governing set of partial differential equations now have coefficients that are functions of $\xi$ and $T$ only. These equations are

$$
\begin{gathered}
\frac{\partial \omega_{r}}{\partial T}+\frac{i n \Gamma_{0} \psi_{1}^{2}}{\xi^{2}} \omega_{r}=-\frac{\epsilon}{2} \omega_{r}, \\
\frac{\partial \omega_{\theta}}{\partial T}+\frac{i n \Gamma_{0} \psi_{1}^{2}}{\xi^{2}} \omega_{\theta}=-\frac{\epsilon}{2} \omega_{\theta}-\frac{2 \Gamma_{0} \psi_{1}^{2}}{\xi^{2}} \omega_{r},
\end{gathered}
$$

and

$$
\frac{\partial \omega_{z}}{\partial T}+\frac{i n \Gamma_{0} \psi_{1}^{2}}{\xi^{2}} \omega_{z}=\epsilon \omega_{z}
$$

The equations for $\omega_{r}$ and $\omega_{z}$ are uncoupled and can be solved directly, while the equation for $\omega_{\theta}$ depends upon the solution for $\omega_{r}$. On solving (12)-(14), it is found that

$$
\begin{gathered}
\omega_{r}=\omega_{r}^{0}(\xi, \zeta) \psi_{1} \psi_{2} E \\
\omega_{\theta}=\left[\omega_{\theta}^{0}(\xi, \zeta)-\frac{2 \Gamma_{0}}{\xi^{2}} \int_{0}^{T} \psi_{1}^{2}\left(T^{\prime}\right) d T^{\prime} \omega_{r}^{0}(\xi, \zeta)\right] \psi_{1} \psi_{2} E,
\end{gathered}
$$

and

$$
\omega_{z}=\omega_{z}^{0}(\xi, \zeta) \psi_{1}^{2} E
$$

where $\omega_{r}^{0}=\omega_{r}, \omega_{\theta}^{0}=\omega_{\theta}$, and $\omega_{z}^{0}=\omega_{z}$ at $T=0$. The exponential factor $E$ is defined as

$$
E(\xi, T)=\exp \left(-\frac{i n \Gamma_{0}}{\xi^{2}} \int_{0}^{T} \psi_{1}^{2}\left(T^{\prime}\right) d T^{\prime}\right)
$$

In all cases, $|E|=1$ and hence the factor $E$ in no way contributes to the growth or decay of the amplitude of any quantity. It should be noted at this point, that if the straining parameter $\epsilon$ is a positive constant, then the radial and tangential vorticity components will decay in time, while the maximum of the axial vorticity component will grow in time, with the radial location of this maximum approaching the core. This is certainly not surprising but it is the effects of this behaviour on the velocity field that will be of interest.

The solutions (15)-(17) provide the means to describe the evolution of an arbitrary disturbance to a potential vortex under strain. In order to do this, it is only necessary to prescribe 
initial conditions in terms of the vorticity components subject to the constraint that the vorticity vector must be solenoidal. Thus, only two of the three components $\omega_{r}^{0}(\xi, \zeta), \omega_{\theta}^{0}(\xi, \zeta)$, and

$\omega_{z}^{0}(\xi, \zeta)$ are independent. At time $T=0$, for example, two components are specified while the third is found from the relation

$$
\frac{1}{\xi} \frac{\partial\left(\xi \omega_{r}^{0}\right)}{\partial \xi}+\frac{i n}{\xi} \omega_{\theta}^{0}+\frac{\partial \omega_{z}^{0}}{\partial \zeta}=0
$$

Once the vorticity has been determined, the perturbation velocities $\vec{u}=\left(u_{r}, v_{\theta}, w\right)$ are derived by integrating the equations obtained from the definitions of the vorticity, namely

$$
\begin{aligned}
& \omega_{r}=\frac{i n \psi_{1}}{\xi} w-\psi_{2} \frac{\partial v_{\theta}}{\partial \zeta}, \\
& \omega_{\theta}=\psi_{2} \frac{\partial u_{r}}{\partial \zeta}-\psi_{1} \frac{\partial w}{\partial \xi},
\end{aligned}
$$

and

$$
\omega_{z}=\frac{\psi_{1}}{\xi}\left(\frac{\partial\left(\xi v_{\theta}\right)}{\partial \xi}-i n u_{r}\right)
$$

The homogeneous solution to (20)-(22) represents the potential velocity field and is included in the integration in order to satisfy velocity boundary conditions.

\section{INITIAL PERTURBATIONS}

In this section, various initial conditions are examined in order to highlight the dynamics of the flow with special attention given to the evolution of the velocity field. This is especially relevant in view of the fact that boundary conditions are in terms of the velocities rather than the vorticity. The actual solving of the partial differential equations for the velocity components can be avoided by using a Fourier transform in the $\zeta$ variable without loss of generality. All dependent quantities can be transformed as

$$
\hat{f}(\xi, \gamma)=\int_{-\infty}^{\infty} f(\xi, \zeta) e^{i \gamma \zeta} d \zeta
$$

It is noted that only in the moving coordinate system will the Fourier transform provide the simplifications that allow the analytical solution to progress. Equivalently, separable solutions in the moving coordinate can be sought, and this approach is taken here. Of course, initially twodimensional disturbances can be considered and should the initial disturbance be independent of the $z$-variable, for example, then for each $n$ the governing equations are ordinary differential equations at the outset and the transform is not required.

\subsection{Impulsive Sources}

The first case considered is the two-dimensional disturbance with sources specified mathematically as Dirac delta functions in terms of the radial variable. This choice essentially provides a Green's function for all arbitrary disturbances in this class. At time $t=0$ and in the physical coordinates, the vorticity components are taken as

$$
\omega_{z}^{0}=\Omega_{z} \delta\left(r-r_{0}\right), \quad \omega_{\theta}^{0}=\Omega_{\theta} \delta^{\prime}\left(r-r_{0}\right),
$$


and, for consistency, it is required that

$$
\omega_{r}^{0}=-i n \Omega_{\theta} \frac{\delta\left(r-r_{0}\right)}{r} .
$$

The full evolution of the vorticity is readily discerned from equations (15)-(17) while the axial velocity is found from (20) to be simply

$$
w=-\Omega_{\theta} \delta\left(\xi-r_{0}\right) \psi_{2} E(\xi, T) .
$$

The radial velocity must satisfy

$$
\xi^{2} \frac{d^{2} u_{r}}{d \xi^{2}}+3 \xi \frac{d u_{r}}{d \xi}+\left(1-n^{2}\right) u_{r}=-i n \Omega_{z} \xi \delta\left(\xi-r_{0}\right) \psi_{1} E(\xi, T)
$$

which has the solution (for $n>0$; analogous solutions for $n<0$ )

$$
u_{r}=\frac{i \Omega_{z} \psi_{1} E\left(r_{0}, T\right)}{2}\left\{\begin{array}{cc}
\left(\frac{\xi}{r_{0}}\right)^{n-1} & \xi<r_{0} \\
\left(\frac{\xi}{r_{0}}\right)^{-n-1} & \xi>r_{0}
\end{array} .\right.
$$

The corresponding tangential velocity is

$$
v_{\theta}=\frac{\Omega_{z} \psi_{1} E\left(r_{0}, T\right)}{2}\left\{\begin{array}{ll}
-\left(\frac{\xi}{r_{0}}\right)^{n-1} & \xi<r_{0} \\
\left(\frac{\xi}{r_{0}}\right)^{-n-1} & \xi>r_{0}
\end{array} .\right.
$$

To interpret the results, the velocities are transformed back to the physical coordinates $r$ and $t$ and the factor $e^{i n \theta}$ is included. It might also be of interest to calculate another physical quantity, such as energy. For this example, both the velocities and the energy are determined.

To highlight the differences of the strained vortex as opposed to one in the unstrained state, both $\epsilon=0$ and $\epsilon=\epsilon_{0}$, a constant, are considered. For the unstrained vortex, $\epsilon=0$, $\psi_{1}=\psi_{2}=1$, and in physical coordinates, $\hat{E}(r, t)=E(r, t) e^{i n \theta}=\exp \left(i n\left(\theta-\frac{\Gamma_{0}}{r^{2}} t\right)\right)$ with the velocity components becoming

$$
\begin{gathered}
w=-\Omega_{\theta} \delta\left(r-r_{0}\right) \hat{E}(r, t), \\
u_{r}=\frac{i \Omega_{z} \hat{E}\left(r_{0}, t\right)}{2} \begin{cases}\left(\frac{r}{r_{0}}\right)^{n-1} & r<r_{0} \\
\left(\frac{r}{r_{0}}\right)^{-n-1} & r>r_{0}\end{cases}
\end{gathered}
$$

and

$$
v_{\theta}=\frac{\Omega_{z} \hat{E}\left(r_{0}, t\right)}{2}\left\{\begin{array}{ll}
-\left(\frac{r}{r_{0}}\right)^{n-1} & r<r_{0} \\
\left(\frac{r}{r_{0}}\right)^{-n-1} & r>r_{0}
\end{array} .\right.
$$

It is noted that the velocity has a traveling wave solution in the angular direction.

For constant straining, $\epsilon=\epsilon_{0}, \psi_{1}=\exp \left(\frac{\epsilon_{0}}{2} t\right), \psi_{2}=\exp \left(-\epsilon_{0} t\right)$ and in moving coordinates $\hat{E}(\xi, t)=E(\xi, t) e^{i n \theta}=\exp \left(i n\left(\theta-\frac{\Gamma_{0}}{\epsilon_{0} \xi^{2}}\left(e^{\epsilon_{0} t}-1\right)\right)\right)$. The resulting velocity components in physical coordinates are

$$
\begin{gathered}
w=-\Omega_{\theta} \delta\left(r e^{\frac{\epsilon_{0}}{2} t}-r_{0}\right) \exp \left(-\epsilon_{0} t\right) \hat{E}\left(r \psi_{1}, t\right), \\
u_{r}=\frac{i \Omega_{z} \hat{E}\left(r_{0}, t\right)}{2}\left\{\begin{array}{cc}
e^{\frac{n \epsilon_{0}}{2}}\left(\frac{r}{r_{0}}\right)^{n-1} & r<r_{0} e^{-\frac{\epsilon_{0} t}{2}} \\
e^{-\frac{n \epsilon_{0}}{2} t}\left(\frac{r}{r_{0}}\right)^{-n-1} & r>r_{0} e^{-\frac{\epsilon_{0} t}{2}}
\end{array},\right.
\end{gathered}
$$


and

$$
v_{\theta}=\frac{\Omega_{z} \hat{E}\left(r_{0}, t\right)}{2}\left\{\begin{array}{ll}
-e^{\frac{n \epsilon_{0}}{2} t}\left(\frac{r}{r_{0}}\right)^{n-1} & r<r_{0} e^{-\frac{\epsilon_{0} t}{2}} \\
e^{-\frac{n \epsilon_{0}}{2} t}\left(\frac{r}{r_{0}}\right)^{-n-1} & r>r_{0} e^{-\frac{\epsilon_{0} t}{2}}
\end{array} .\right.
$$

For this case, there is a squeezing effect in which the overall pattern collapses into a core, the size of which is decaying exponentially in time. When $\Omega_{\theta} \neq 0$ and $\Omega_{z}=0$, the magnitude of the velocity decays and the traveling wave motion, described by the quantity $\hat{E}$, decelerates. For $\Omega_{z} \neq 0$ and $\Omega_{\theta}=0$, there is an apparent exponential growth in the magnitude of the velocities $u_{r}$ and $v_{\theta}$ in the developing core. This is seen by noting that the velocities have their maximum values at $\xi=r_{0}$ (or $r=r_{0} e^{-\frac{\epsilon_{0} t}{2}}$ ) and the maximum values grow as $e^{\frac{\epsilon_{0} t}{2}}$. There is also a corresponding exponential increase in the velocity of the traveling wave. Thus, the two modes are seen to behave differently. It is unclear how these effects relate to the overall stability of the flow.

To explore the dynamics from a different perspective, the disturbance energy density, given by

$$
\mathcal{E}_{n}=\frac{1}{2} \int_{0}^{\infty}\left(\left|u_{r}\right|^{2}+\left|v_{\theta}\right|^{2}+|w|^{2}\right) r d r
$$

is conveniently calculated in the moving coordinates by

$$
\mathcal{E}_{n}=\frac{1}{2 \psi_{1}^{2}} \int_{0}^{\infty}\left(\left|u_{r}\right|^{2}+\left|v_{\theta}\right|^{2}+|w|^{2}\right) \xi d \xi
$$

By considering only the case $\Omega_{z} \neq 0$ and $\Omega_{\theta}=0$, the energy is

$$
\mathcal{E}_{n}=\frac{\Omega_{z}^{2} r_{0}^{2}}{4|n|}
$$

so that the perturbation energy for these two-dimensional disturbances remains constant during the entire evolution even though the velocity profiles are increasing in magnitude. This implies that the long time behaviour predicted by the Green's function will most certainly be modified when considering a spatially distributed source of vorticity. In the next subsection, this conjecture is shown to be true.

Another set of initial conditions for which analytic solutions can be determined is taken as being axisymmetric $(n=0)$ with normal mode behaviour in the axial direction. Again, using Dirac delta functions to facilitate analytic solutions, the initial vorticity is specified as

$$
\omega_{r}^{0}=\Omega_{r} \delta\left(r-r_{0}\right) e^{-i \gamma_{0} z}
$$

and

$$
\omega_{\theta}^{0}=\Omega_{\theta} \delta^{\prime}\left(r-r_{0}\right) e^{-i \gamma_{0} z} .
$$

The value of $\omega_{z}^{0}$, which is not needed in order to determine the velocities, is determined by solving (19). The vorticity solutions of the initial value problem are given by equations (15)-(17) with the exponential factor $E=1$. The angular velocity $v_{\theta}$ is found to be

$$
v_{\theta}=\frac{-i \Omega_{r}}{\gamma_{0}} \psi_{1} e^{-i \gamma_{0} \zeta} \delta\left(\xi-r_{0}\right)
$$


To determine the radial and axial velocity components, it is noted that the solutions are separable in $\xi$ and $\zeta$. Hence, by dropping the exponential factor $e^{-i \gamma_{0} \zeta}$ in all quantities, the ordinary differential equations that the remaining velocities must satisfy are

$$
\xi^{2} \frac{d^{2} u_{r}}{d \xi^{2}}+\xi \frac{d u_{r}}{d \xi}-\left[1+\frac{\gamma_{0}^{2} \psi_{2}^{2}}{\psi_{1}^{2}} \xi^{2}\right] u_{r}=\frac{-i \gamma_{0} \psi_{2}^{2}}{\psi_{1}} \xi^{2}\left[\Omega_{\theta}-\Omega_{r} \frac{2 \Gamma_{0}}{\xi^{2}} \int_{0}^{T} \psi_{1}^{2} d T^{\prime}\right] \delta\left(\xi-r_{0}\right)
$$

and

$$
w=\frac{\psi_{1}}{i \gamma_{0} \psi_{2}} \frac{d}{d \xi}\left(\xi u_{r}\right)
$$

The solutions of (42) and (43) are

$$
u_{r}=\frac{-i \gamma_{0} r_{0} \psi_{2}^{2}}{\psi_{1}}\left[\Omega_{\theta}-\Omega_{r} \frac{2 \Gamma_{0}}{r_{0}^{2}} \int_{0}^{T} \psi_{1}^{2} d T^{\prime}\right] \begin{cases}K_{1}\left(\frac{\psi_{2}}{\psi_{1}}\left|\gamma_{0}\right| r_{0}\right) I_{1}\left(\frac{\psi_{2}}{\psi_{1}}\left|\gamma_{0}\right| \xi\right) & \xi<r_{0} \\ K_{1}\left(\frac{\psi_{2}}{\psi_{1}}\left|\gamma_{0}\right| \xi\right) I_{1}\left(\frac{\psi_{2}}{\psi_{1}}\left|\gamma_{0}\right| r_{0}\right) & \xi>r_{0}\end{cases}
$$

and

$$
w=\frac{-r_{0} \psi_{2}^{2}\left|\gamma_{0}\right|}{\psi_{1}}\left[\Omega_{\theta}-\Omega_{r} \frac{2 \Gamma_{0}}{r_{0}^{2}} \int_{0}^{T} \psi_{1}^{2} d T^{\prime}\right] \begin{cases}K_{1}\left(\frac{\psi_{2}}{\psi_{1}}\left|\gamma_{0}\right| r_{0}\right) I_{0}\left(\frac{\psi_{2}}{\psi_{1}}\left|\gamma_{0}\right| \xi\right) & \xi<r_{0} \\ K_{0}\left(\frac{\psi_{2}}{\psi_{1}}\left|\gamma_{0}\right| \xi\right) I_{1}\left(\frac{\psi_{2}}{\psi_{1}}\left|\gamma_{0}\right| r_{0}\right) & \xi>r_{0}\end{cases}
$$

where $K_{1}$ and $I_{1}$ are the modified Bessel functions.

On examining the radial and axial velocities, it is found that in the absence of a straining vortex field, the terms proportional to $\Omega_{\theta}$ are stationary in time whereas the magnitudes of the terms proportional to $\Omega_{r}$ grow linearly in $T$ for all time. The addition of strain to the vortex field has a stabilizing effect in the following manner. The terms proportional to $\Omega_{\theta}$ are always exponential in time and decay in magnitude. The terms proportional to $\Omega_{r}$ display algebraic growth for small time and the large time behaviour under constant strain, namely

$$
u_{r}=\Omega_{r} \frac{2 i \gamma_{0} \Gamma_{0}}{\epsilon_{0} r_{0}} e^{-\epsilon_{0} t}\left\{\begin{array}{cl}
\frac{r}{r_{0}} & r<r_{0} e^{-\frac{\epsilon_{0}}{2} t} \\
e^{-\epsilon_{0} t \frac{r_{0}}{r}} & r>r_{0} e^{-\frac{\epsilon_{0}}{2} t}
\end{array}\right.
$$

shows eventual exponential decay (the axial velocity component decays even faster). Even though standard stability based on large time behaviour would consider this flow to be stable, the initial algebraic growth could be large enough so that nonlinear effects might become significant before the exponential decay dominates. It is stressed that the solutions are not restricted to constant strain. For example, if $\epsilon(t)$ had the behaviour that $\epsilon(0) \neq 0$ but $\epsilon(t) \rightarrow 0$ for large time, the following scenario is possible: A period of algebraic growth is followed by a period exponential decline, and then the very large time behaviour returns to algebraic growth. Clearly, the overall effects depend upon the relative time scales.

It is of quite some interest to compare the results of the axisymmetric initial disturbance with the two-dimensional initial disturbance. Although the vorticity components evolving from a two-dimensional initial disturbance have linear growth in time (which continues indefinitely for zero strain), the velocity components do not exhibit this behaviour. For this case, the term that produces the linear growth, namely $\int_{0}^{T} \psi_{2}^{2} d t^{\prime}$, only appears in the exponential factor that controls the traveling wave and not in the magnitude of the velocities. In the case of the axisymmetric disturbance, the linear growth term appears directly in the magnitude of the velocities. 


\subsection{Specific Initial Conditions}

In this section, special initial sources of vorticity are employed in order to understand how the vortex evolves in response to more general initial disturbances. Although complete analytical solutions for the velocities can be written in terms of the above Green's functions, the resulting integrals are not easily analyzed. Therefore, numerical integration of the governing ordinary differential equations has been used instead.

First, the two-dimensional disturbance (independent of the axial variable $\zeta$ ) is considered and is taken to be

$$
\begin{gathered}
\omega_{z}^{0}=\Omega_{z} \frac{1}{\sqrt{4 \pi \sigma}} e^{\frac{-\left(r-r_{0}\right)^{2}}{4 \sigma}}, \\
\omega_{\theta}^{0}=-\Omega_{\theta} \frac{r-r_{0}}{2 \sigma \sqrt{4 \pi \sigma}} e^{\frac{-\left(r-r_{0}\right)^{2}}{4 \sigma}},
\end{gathered}
$$

and

$$
\omega_{r}^{0}=i n \Omega_{\theta} \frac{e^{\frac{-r_{0}^{2}}{4 \sigma}}-e^{\frac{-\left(r-r_{0}\right)^{2}}{4 \sigma}}}{r \sqrt{4 \pi \sigma}} .
$$

These sources of vorticity are chosen such that, as $\sigma \rightarrow 0$, the above functions reduce to the Dirac delta function and its derivative centered at $r_{0}$ and compare directly to the initial conditions (24), and (25). The velocities must satisfy

$$
\begin{gathered}
w=\Omega_{\theta} \frac{e^{\frac{-r_{0}^{2}}{4 \sigma}}-e^{\frac{-\left(\xi-r_{0}\right)^{2}}{4 \sigma}}}{\xi^{2} \sqrt{4 \pi \sigma}} \psi_{2} E(\xi, T), \\
\xi^{2}\left(u_{r}\right)_{\xi \xi}+3 \xi\left(u_{r}\right)_{\xi}+\left(1-n^{2}\right) u_{r}=-\frac{i n \xi \psi_{1} \Omega_{z}}{\sqrt{4 \pi \sigma}} e^{\frac{-\left(\xi-r_{0}\right)^{2}}{4 \sigma}} E(\xi, T),
\end{gathered}
$$

and

$$
\xi^{2}\left(v_{\theta}\right)_{\xi \xi}+3 \xi\left(v_{\theta}\right)_{\xi}+\left(1-n^{2}\right) v_{\theta}=\frac{\psi_{1} \Omega_{z}}{\sqrt{4 \pi \sigma}} \frac{d}{d \xi}\left(\xi^{2} e^{\frac{-\left(\xi-r_{0}\right)^{2}}{4 \sigma}} E(\xi, T) .\right)
$$

Equation (51) was solved numerically for various values of $n$ and $\epsilon_{0}$ with $\sigma=.5$, and $\Omega_{z}=1.0$. The results are shown in Figures 1 and 2 as functions of the real physical variables $r$ and $t$. Figure 1 gives the maximum magnitude of the velocity component $u_{r}$ as a function of time. Contrary to the prediction of the analytic solution of the Green's function, the large time behaviour of this maximum is decay rather than exponential growth. The initial behaviour, on the other hand, does show transient growth that is dependent on the strain rate. Thus, for large strain rates, finite amplitude disturbances may cause the system to become nonlinear, even though the long-time solution is stable. In Figure 2, the velocity profiles for $u_{r}$ are shown for $n=2$ and $\epsilon_{0}=1$. Figures $2 \mathrm{a}-\mathrm{b}$ are for an initial vorticity disturbance which is distributed in space $(\sigma=.5)$. This solution is equivalent to an integration of the Green's function over $r_{0}$, and produces a velocity profile that is decaying in time. Also to be noted is the oscillations as the disturbance is convected into the core by the positive strain. The origin of these fluctuations can

be traced to the exponential factor $\hat{E}(\xi, t)$, and specifically it is a result of the spatial variation in the velocity of the traveling wave in the angular direction. It is interesting to note that the 

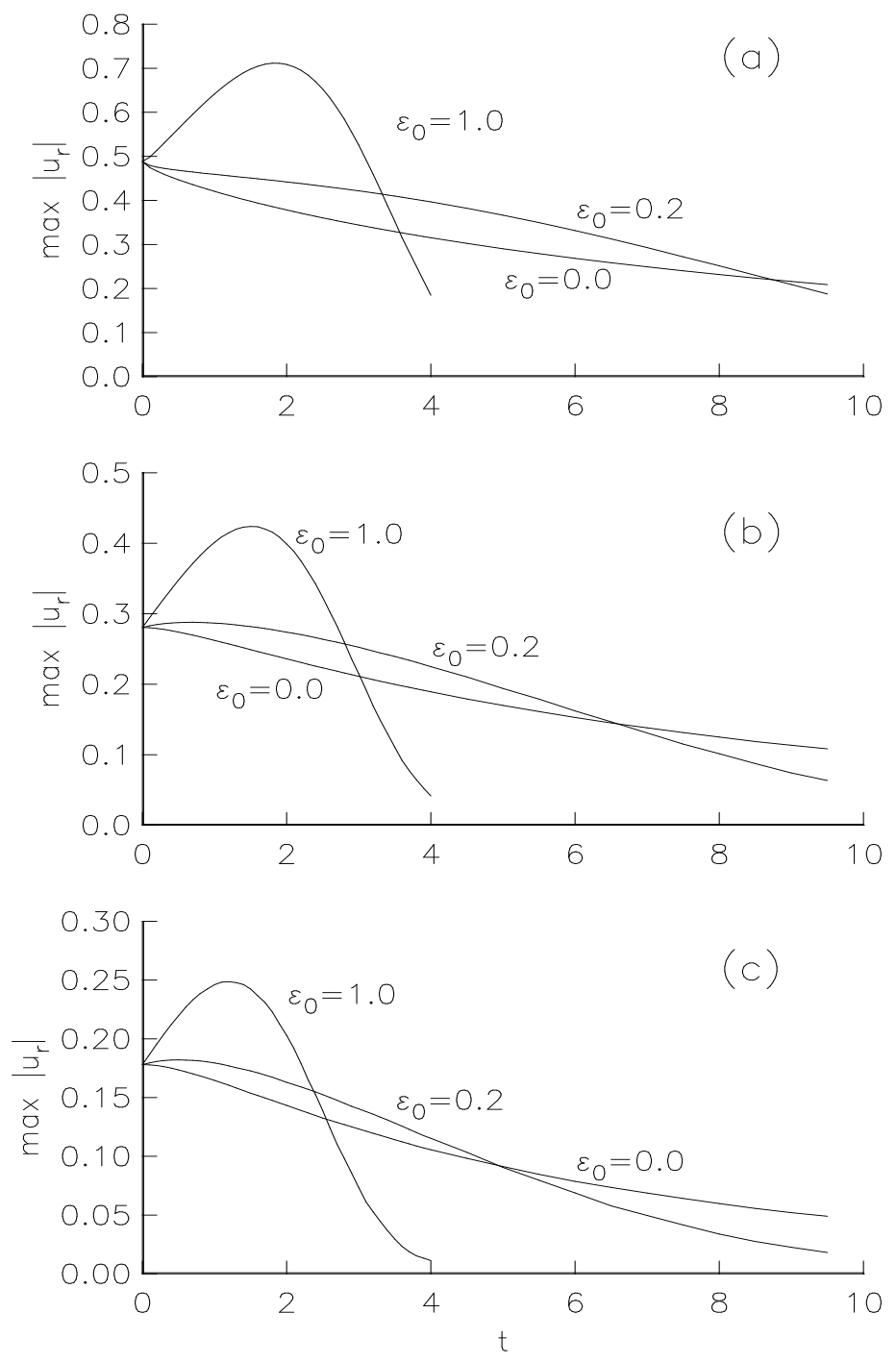

Figure 1: Plot of $\max \left|u_{r}\right|$ as a function of time for a spatially distributed initial source of vorticity $\left(\Omega_{z}=1.0, \Omega_{\theta}=0, \sigma=.5, \Gamma_{0}=1.0\right.$ and $\left.r_{0}=2.0\right)$. Graphs for three values of $n$ are shown: (a) $n=1$, (b) $n=2$, and (c) $n=4$. 

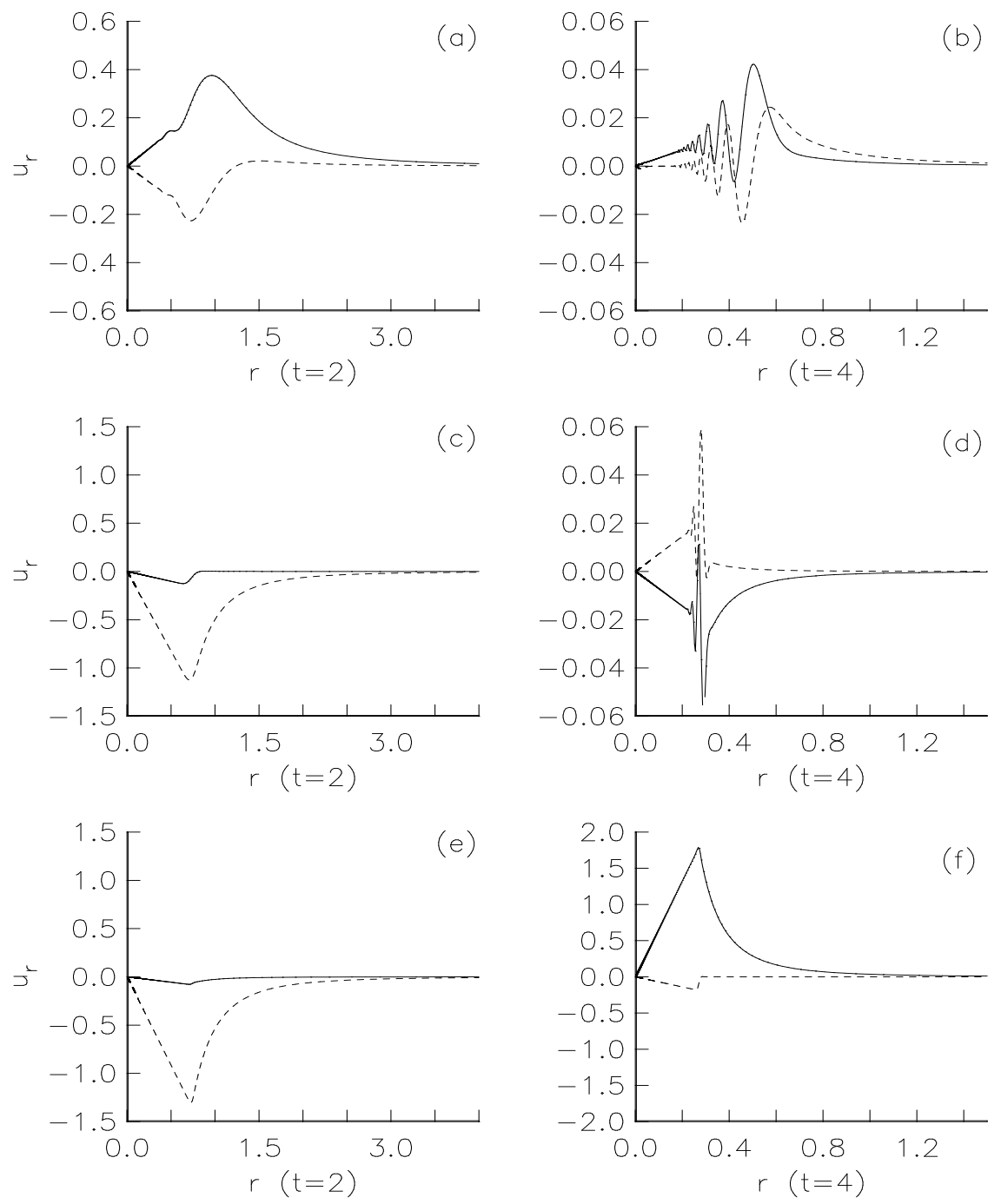

Figure 2: Plot of $\operatorname{Re}\left(u_{r}\right)$ (solid) and $\operatorname{Im}\left(u_{r}\right)$ (dashed) as a function of the physical coordinate $r$ for spatially compact and spatially distributed initial sources of vorticity $\left(\Omega_{z}=1.0, \Omega_{\theta}=0\right.$, $\epsilon=1.0, n=2, \Gamma_{0}=1.0$ and $r_{0}=2.0$ ). Graphs for three values of $\sigma$ are shown: (a)-(b) $\sigma=.5$, (c)-(d) $\sigma=.01$, and (e)-(f) $\sigma=.001$. 

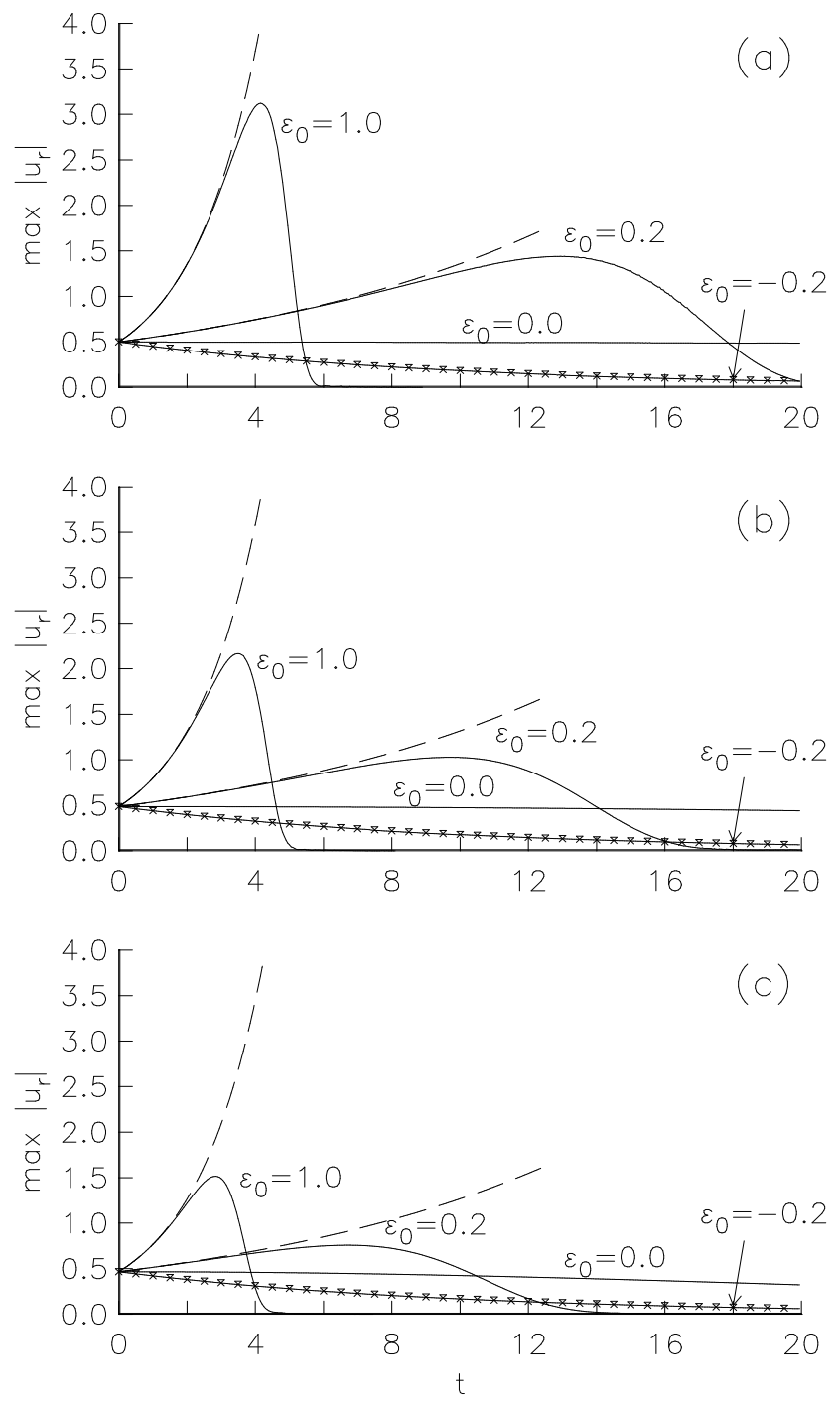

Figure 3: Plot of $\max \left|u_{r}\right|$ as a function of time for a spatially compact initial source of vorticity $\left(\Omega_{z}=1.0, \Omega_{\theta}=0, \sigma=.001, \Gamma_{0}=1.0\right.$ and $\left.r_{0}=2.0\right)$. Graphs for three values of $n$ are shown: (a) $n=1$, (b) $n=2$, and (c) $n=4$. The dashed curves are the Green's function predictions. The symbols and the line through the symbols are the Green's function prediction and the calculated response, respectively, for $\epsilon_{0}=-.2$ 
Green's function does not predict this behaviour directly since the exponential factor appears as $\hat{E}\left(r_{0}, t\right)$. Therefore, the traveling wave velocity has no radial dependence. The velocity fluctuations and general decay in magnitude must be the result of the distributed nature of the initial source itself. To explore this phenomenon, Figures 2c-d and Figures 2e-f show the results of a numerical integration for $\sigma=.01$ and $\sigma=.001$, respectively. Both approximate the solution to a delta function source quite nicely for small times, as can be seen by examining the $t=2$ graphs. The $u_{r}$ profile is linear near the origin and decays for $r$ large as predicted by equation (34). The predicted response to the delta function initial condition continues until the cumulative effects of the exponential factor show themselves at $t=4$. By comparison with the previous results, the rapid fluctuations are concentrated near the peak when $\sigma=.01$ as opposed to the broader fluctuation when $\sigma=.5$ and are not yet seen for $\sigma=.001$. Of particular note is that the magnitude of the solution for $\sigma=.01$ is decaying which is consistent with the prior results for the distributed source, and for the $\sigma=.001$, the magnitude is still growing at $t=4$ which is consistent with the Green's function results. The Green's function predicts exponential growth for all time, but since there is a little bit of spatial variation in the initial profile, the $\sigma=.001$ solution eventually goes the way of the $\sigma=.01$ solution. This can be shown to be the case by examining the maximum of $u_{r}$ as a function of time for $\sigma=.001$. The result of this calculation for the same values of $n$ and $\epsilon_{0}$ as in Figure 1 are shown in Figure 3. For each case with $\epsilon_{0}>0$ there is an initial growth of the maximum, but then eventual decay. The decay is delayed for smaller values of $\epsilon_{0}$ which is consistent with the disturbance entering the core of the vortex as the source of the departure from the Green's function predicted behaviour. Shown as dashed lines in Figure 3 is the growth predicted by the Green's function solution. Of some interest is the behaviour for negative strain. Also shown in Figure 3 is the solution for $\epsilon_{0}=-.2$ which decays and shows no initial growth. In this scenario, the initial disturbance is convected away from the core by the straining, and the Green's function prediction shown as symbols is accurate. This investigation shows that the source of the fluctuations in velocity as the strain convects the disturbance toward the core are indeed a result of the spatial distribution of the initial source of vorticity and that this spatial distribution leads to the eventual decay of perturbations.

The set of initial conditions corresponding to the axisymmetric modes $(n=0)$ are taken as

$$
\begin{aligned}
& \omega_{r}^{0}=\Omega_{r} \frac{r}{r_{0} \sqrt{4 \pi \sigma}} e^{\frac{-\left(r-r_{0}\right)^{2}}{4 \sigma}} e^{-i \gamma_{0} z}, \\
& \omega_{\theta}^{0}=\Omega_{\theta} \frac{r-r_{0}}{2 \sigma \sqrt{4 \pi \sigma}} e^{\frac{-\left(r-r_{0}\right)^{2}}{4 \sigma}} e^{-i \gamma_{0} z},
\end{aligned}
$$

with $\omega_{z}^{0}$ obtained by requiring the vorticity to be solenoidal. The velocities satisfy

$$
v_{\theta}=-\frac{i \Omega_{r} \xi}{\gamma_{0} r_{0} \sqrt{4 \pi \sigma}} e^{\frac{-\left(\xi-r_{0}\right)^{2}}{4 \sigma}} \psi_{1} e^{-i \gamma_{0} \zeta}
$$

and

$\xi^{2} \frac{d^{2} u_{r}}{d \xi^{2}}+\xi \frac{d u_{r}}{d \xi}-\left[1+\frac{\gamma_{0}^{2} \psi_{2}^{2}}{\psi_{1}^{2}} \xi^{2}\right] u_{r}=\frac{-i \gamma_{0} \psi_{2}^{2}}{\psi_{1}} \xi^{2} \frac{1}{\sqrt{4 \pi \sigma}}\left[\Omega_{\theta} \frac{\xi-r_{0}}{2 \sigma}-\frac{2 \Gamma_{0} \Omega_{r}}{r_{0} \xi} \int_{0}^{T} \psi_{1}^{2} d T^{\prime}\right] e^{\frac{-\left(\xi-r_{0}\right)^{2}}{4 \sigma}}$ 


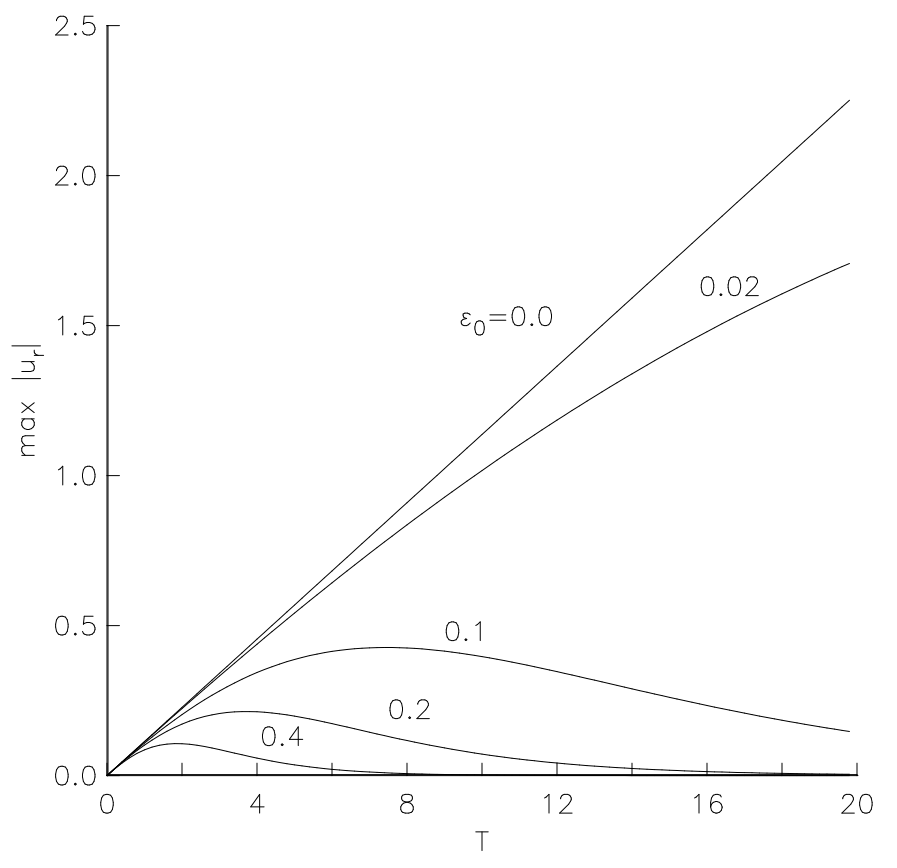

Figure 4: Plot of $\max \left|u_{r}\right|$ as a function of time for an axisymmetric $(n=0)$, spatially distributed initial source of vorticity with $\Omega_{r}=1.0, \Omega_{\theta}=0, \Gamma_{0}=1.0, \sigma=.5, \gamma_{0}=1.0$ and $r_{0}=2.0$. 
with the last equation being integrated numerically. The maximum of $u_{r}$ as a function of time is shown in Figure 4 for various values of the strain rate. For this figure, $\Omega_{\theta}=0$ and $\Omega_{r}=1$. The above equation shows linear growth of the perturbation for the unstrained vortex $\epsilon_{0}=0$. For the strained vortex the same rate of linear growth is initially present and is independent of the choice of $\epsilon_{0}$. However, as time progresses the exponential decay becomes evident with the decay occuring earlier for larger values of strain. The spatial profiles of the velocity are swept toward the center of the vortex by positive straining but, due to the absence of the traveling wave, the profiles remain smooth. For this case where the factor $\hat{E}=1$ and thus is not involved in an integral representation of the solution, the results of the Green's function are predictive, with no further interpretation necessary.

\subsection{Three Dimensional Disturbances}

It has been noted that the quantity $\int_{0}^{T} \psi_{1}^{2} d t^{\prime}$ plays a key role in the dynamics of twodimensional disturbances (independent of the axial variable) and in the dynamics of axisymmetric disturbances. In the first instance this quantity controlled the speed of a traveling wave through the quantity $E e^{i n \theta}$ and noticeable effects are produced when considering a velocity profile that evolves from a spatially distributed initial source. In the second instance, the quantity $\int_{0}^{T} \psi_{1}^{2} d t^{\prime}$ controlled the initial algebraic growth of the amplitude of the radial velocity compo-

nent. For the fully three-dimensional disturbance, the relevant quantity should be $E e^{i n \theta-i \gamma_{0} \zeta}$, which is a helical wave moving along the axial core.

The form of the initial condition selected is given by (53) and (54) with the additional factor of $e^{i n \theta}$. The vorticity is given by (15)-(17) after requiring the initial vorticity to be solenoidal in order to determine the $\omega_{z}$ component. It is convenient to work with the equation for angular velocity given by

$$
\xi^{2} \frac{d v_{\theta}}{d \xi^{2}}+3 \xi \frac{d v_{\theta}}{d \xi}+\left[1-n^{2}-\gamma_{0}^{2} \frac{\psi_{2}^{2}}{\psi_{1}^{2}} \xi^{2}\right] v_{\theta}=\frac{1}{\psi_{1}} \frac{d\left(\xi^{2} \omega_{z}\right)}{d \xi}+i \gamma_{0} \frac{\psi_{2}}{\psi_{1}^{2}} \xi^{2} \omega_{r}
$$

and the other velocity profiles are determined directly from the vorticity equations (20) and (22). The results of the numerical integration for the fully three-dimensional disturbances indeed show the artifacts from the previous special case analysis, but should be considered as a completely different class of solutions. In Figure 5, the amplitudes of the velocity are shown as a function of time for $n=2, r_{0}=4, \gamma_{0}=1, \Omega_{r}=1.0$ and $\omega_{\theta}=0.0$. The dashed curves correspond to a delta-like initial condition with $\sigma=.01$ and the solid curves show the evolution of a spatially distributed initial condition with $\sigma=.2$. The radial velocity demonstrates exponential growth until the disturbance reaches the core of the vortex, and then there is rapid decline. As before, the spatially compact initial vorticity disturbance experiences the exponential growth for longer periods of time. The results for the other two velocity components are a little more ambiguous. For the tangential velocity, there appears to be a weaker exponential growth, especially for $\sigma=.01$. For $\sigma=.2$, there is very little growth of the tangential velocity. The axial velocity demonstrates basic exponential decay except for a short initial period where algebraic growth occurs. This is easily seen in Figure $5 \mathrm{c}$ where $\epsilon_{0}=1.0$. For $\epsilon_{0}=.2$ there appears to be a balance between the algebraic growth and the slower exponential decay. 

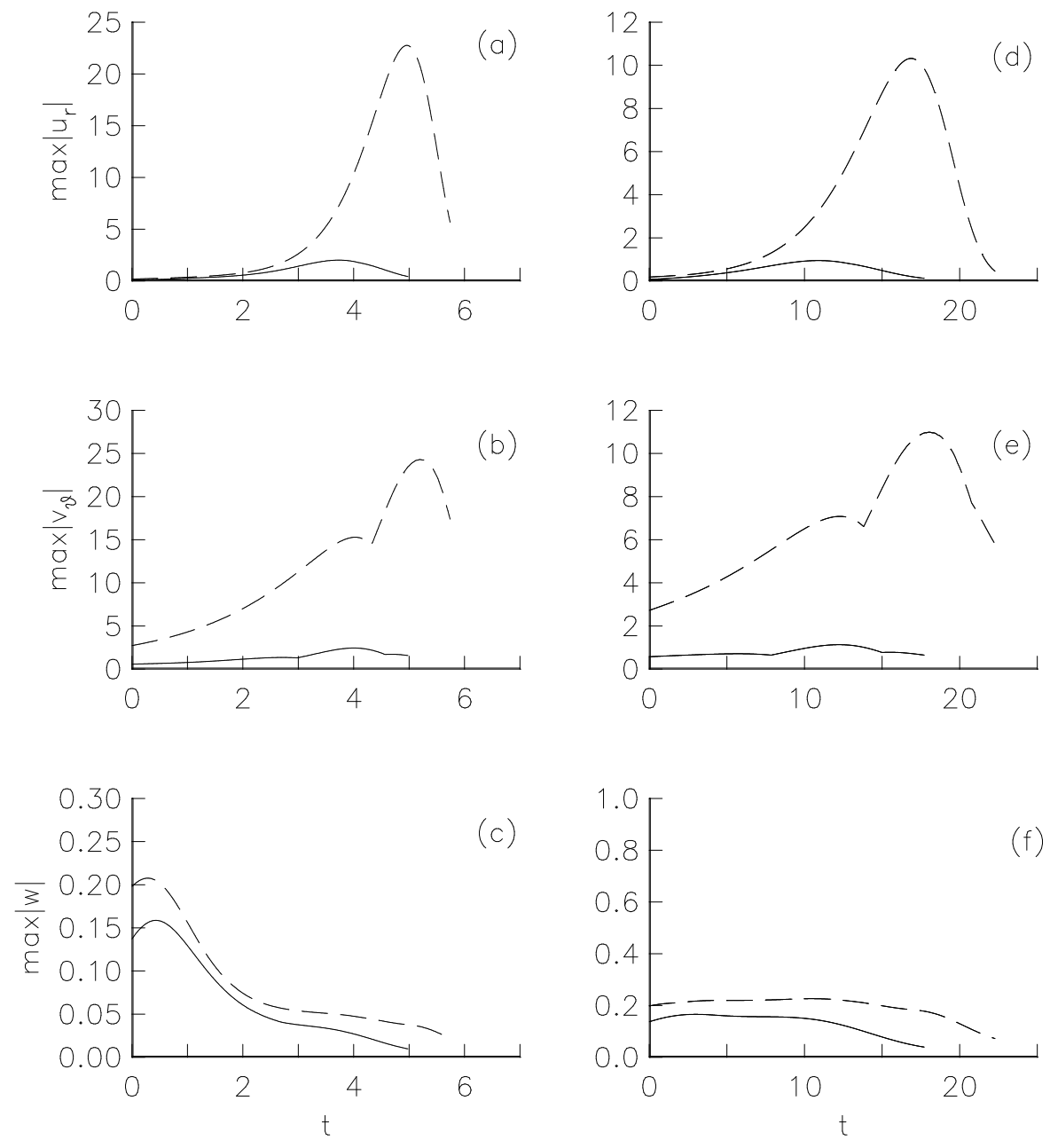

Figure 5: Plot of $\max \left|u_{r}\right|, \max \left|v_{\theta}\right|$ and $\max |w|$ as a function of time for a spatially compact ( $\sigma=.01$, dashed) and spatially distributed $\left(\sigma=.2\right.$, solid) initial source of vorticity $\left(\Omega_{r}=1.0\right.$, $\Omega_{\theta}=0, n=2, \Gamma_{0}=1.0, \gamma_{0}=1.0$, and $\left.r_{0}=4.0\right)$. Two values of the strain rate were chosen: (a-c) $\epsilon_{0}=1.0$ and (d-f) $\epsilon_{0}=0.2$. 

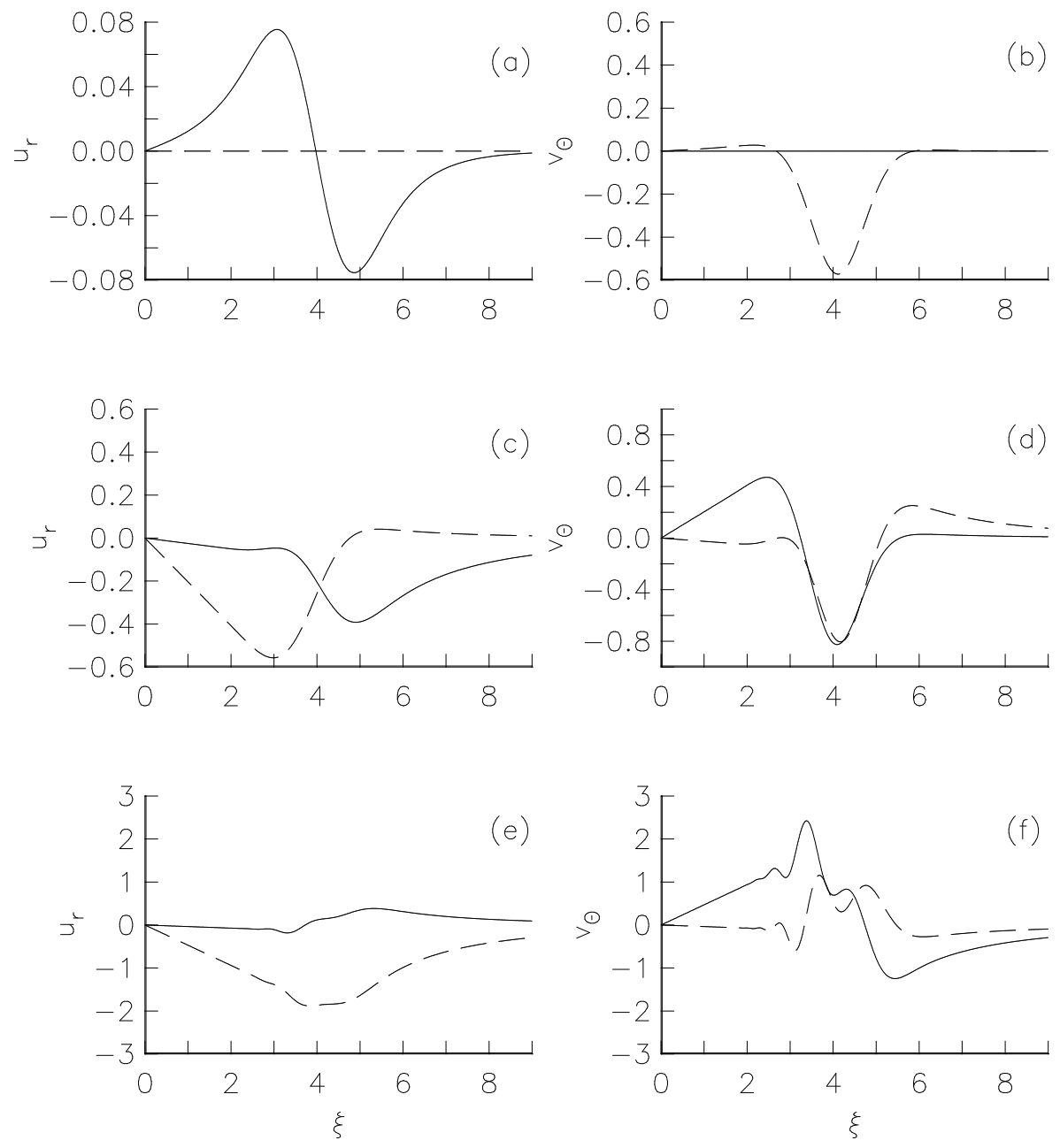

Figure 6: Plot of the real part (solid) and imaginary part (dashed) as a function of the moving coordinate $\xi$ for a spatially distributed initial source of vorticity $\left(\Omega_{r}=1.0, \Omega_{\theta}=0, \sigma=.2\right.$, $\epsilon=1.0, n=2, \Gamma_{0}=1.0$ and $r_{0}=4.0$ ). Graphs for three values of time $t$ are shown: (a)-(b) $t=0$, (c)-(d) $t=2$, and (e)-(f) $t=4$. 

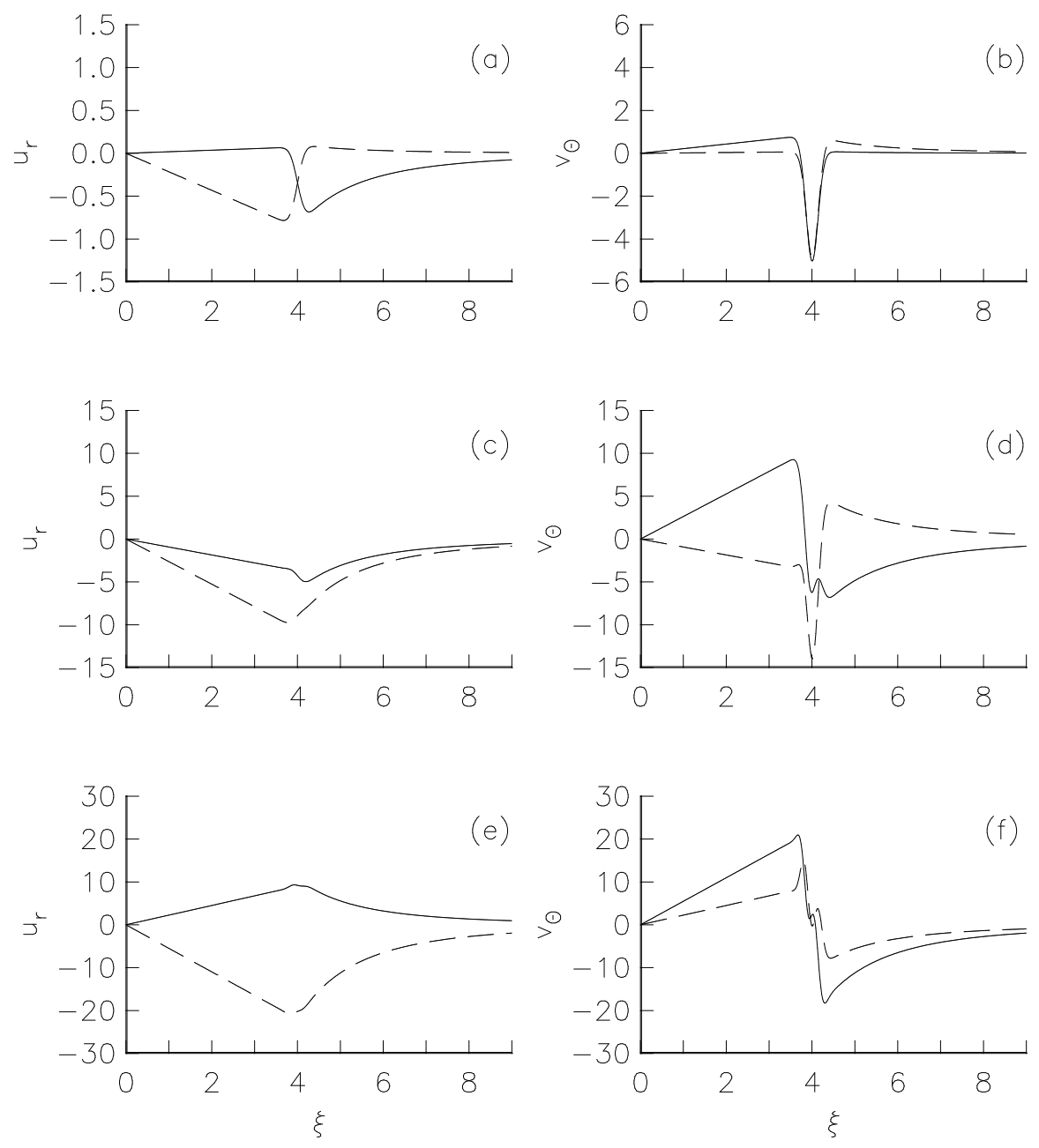

Figure 7: Plot of the real part (solid) and imaginary part (dashed) as a function of the moving coordinate $\xi$ for a spatially compact initial source of vorticity $\left(\Omega_{r}=1.0, \Omega_{\theta}=0, \sigma=.01\right.$, $\epsilon=1.0, n=2, \Gamma_{0}=1.0$ and $r_{0}=4.0$ ). Graphs for three values of time $t$ are shown: (a)-(b) $t=2$, (c)-(d) $t=4$, and (e)-(f) $t=5$. 
In Figures 6 and 7, the angular and radial velocity profiles are shown as functions of the moving coordinate $\xi$ for $\epsilon_{0}=1.0, r_{0}=4.0, n=2, \gamma=1.0 \Omega_{r}=1.0$, and $\Omega_{\theta}=0$ which are the conditions for Figure 5a-c. The magnitudes of the velocities are increasing as time progresses. At around $t=4$, the effects of the factor $E$ start producing the fluctuations in the tangential velocity components with the fluctuations being more prevalent for the spatially distributed initial conditions than for the spatially compact initial conditions. For the case $\sigma=.2$, the radial fluctuations in the radial velocity profile are not as visually evident, but have produced somewhat of a smoothing effect. For the case of $\sigma=.01$ there are no discernible effects of the factor $E$ on the radial velocity profile, even for $t=5$. This is because the effects are still concentrated in a small interval around $\xi=4$ as can be seen by the figures for the tangential velocity. As time progresses, the solutions for $\sigma=.01$ will show greater signs of the effects of the factor $E$ and will decay in magnitude quite rapidly as can be determined from Figure 5 .

\section{CONCLUSIONS}

The general problem of vortices subjected to perturbations is considered within a completely different framework than has heretofore been done. First, the nature of any vortex is reviewed with respect to the velocity field that might be required. Intrinsically, it is suggested that any vortex must be three-dimensional and such flows are usually under the influence of positive or negative strain.

Second, the question of what might happen if perturbations are introduced into one of these flows has been a major challenge due to the fact that no direct mathematical analysis has been possible. The use of classical stability techniques that employ separable solutions is not possible in view of the underlying complications. Indeed, save for some numerical treatments related to special approximations, success has been only with those flows that are vortical in a plane. This work offers a means whereby these obstacles can be overcome and still include the full three-dimensionality of both the mean vortex and the perturbation field.

If the strength of the vortex is strong enough and no boundaries are present, any effects due to viscosity are confined to the core. In effect, this means the core has a finite size and no component of the velocity is discontinuous. To a large degree of approximation, viscous effects can also be neglected in the analysis of the perturbation dynamics. Even on this basis, it is shown that the perturbation field does develop a core and no component of the velocity results in a discontinuity. And, the benefit of being able to obtain analytical closed form solutions for the initial-value, boundary-value problem is an asset. This has been possible for arbitrary initial values. Specifically, a potential three-dimensional vortex is subjected to perturbations at time $\mathrm{t}=0$ and the full three-dimensional problem is solved for all values of time.

The strategy used for solution directly involves the perturbation vorticity. The linearized equations governing the vorticity are solved subject to initial distributions specified as functions of the space variables. The kinematic requirement that the vorticity be solenoidal is insured. Then, these results are used to solve for the velocity and meet proper boundary conditions. For localized vorticity (specified as Dirac delta functions), there is transient growth in the magnitudes of the velocity components. It is also found that the larger the strain rate, the larger the transient

growth and this goes on until the disturbance enters the core of the vortex. For distributions 
of the perturbation vorticity that are more evenly distributed in space, there is little, if any, transient growth for the velocity. Asymptotically, no disturbance survives regardless of whether or not it is localized. The special cases of a two-dimensional or an axisymmetric vortex are included. Even though diffusion is absent in this work, it appears that, once vorticity is diffuse - as can be inferred from the more evenly distributed initial input - then even severe strain cannot enhance the flow.

\section{References}

[1] A.D.D. Craik and W.O. Criminale. Evolution of wavelike disturbances in shear flows: A class of exact solution of the Navier-Stokes equations. Proc. Roy. Soc. London A, 234:613, 1992.

[2] B.J. Bayly. Three-dimensional instability of elliptical flow. Physical Review Letters, 57:21602163, 1986.

[3] M.M. Landman and P.G. Saffman. The three-dimensional instability of strained vortices in a viscous fluid. Phys. Fluids, 30:2339-2342, 1987.

[4] F. Waleffe. On the three-dimensional instabilities of strained vortices. Phys. Fluids A, $2: 76-80,1990$.

[5] D.G. Dritschel. The stability of elliptical vortices in an external straining flow. J. Fluid Mech., 210:223-261, 1990.

[6] A.D.D. Craik and H.R. Allen. The stability of three-dimensional time-periodic flows with spatially uniform strain rates. J. Fluid Mech., 234:613-627, 1991.

[7] C.P. Donaldson and R.O. Sullivan. Examination of the solutions of the Navier-Stokes equations for a class of three-dimensional vortices, part I: Velocity distributions for the steady motion. Technical Report AFOSR TN 60-1227, Aeronautical Research Associates of Princeton, 1960. 